\title{
HSWI/SNF Complex
}

National Cancer Institute

\section{Source}

National Cancer Institute. hSWIISNF Complex. NCI Thesaurus. Code C19837.

A family of ATP-dependent chromatin-remodeling complexes implicated in the regulation of gene expression, cell cycle control, and oncogenesis. These complexes facilitate transcriptional regulation of specific genes by modifying DNA-histone interactions within nucleosomes. 